\title{
Numerical Simulation of Heat Transfer Performance of Calender Roll Structure in Glass Calendering
}

\author{
Geng Tie, Sheng Jie, Hu Jinzhong
}

School of Mechanical and Electrical Engineering, Henan University of Technology, Zhengzhou, China

Email address:

Tiegeng2000@163.com (Geng Tie), 735373933@qq.com (Sheng Jie), 15003882735@163.com (Hu Jinzhong)

To cite this article:

Geng Tie, Sheng Jie, Hu Jinzhong. Numerical Simulation of Heat Transfer Performance of Calender Roll Structure in Glass Calendering. International Journal of Mechanical Engineering and Applications. Vol. 5, No. 2, 2017, pp. 118-128. doi: 10.11648/j.ijmea.20170502.17

Received: March 30, 2017; Accepted: May 5, 2017; Published: May 10, 2017

\begin{abstract}
This paper analyzes the heat transfer characteristics of Heat transfer characteristics during photovoltaic glass calendering, establish the forming process numerical simulation equation, adopt Computational Fluid Dynamics(CFD) numerical simulation method, by introducing reasonable simplification and hypothesis, a mathematical model for the flow and heat transfer of cooling water In different structure calendering roll cavity was established. The effect of the cooling water channel structure on the heat transfer performance of the calender roll was analyzed by FLUENT simulation results.
\end{abstract}

Keywords: Photovoltaic Glass, Calendering, FLUENT, Calendering Roll, Numerical Simulation

\section{Introduction}

With the gradual reduction of non-renewable energy, countries around the world pay more and more attention to the solar photovoltaic industry, which to a large extent led to the development of photovoltaic glass industry. Photovoltaic calendered glass as a high-end products in the glass products, Has a high transmittance and low reflectivity characteristics of the sun, has been widely used in the production of solar photovoltaic cells, used to improve the performance of solar cells $[1,2]$. For a long time, the glass molding process is mainly rely on the producers through repeated debugging and correction can get the production capacity, Photovoltaic glass iscontinuous large-scale production, once the furnace is ignited, the daily consumption is huge, so the cost of repeated debugging on the production line is quite high, the quality of this method is difficult to guarantee, and the product development cycle is long, the product yield is very low, which greatly affected the photovoltaic glass product quality and production costs. Use the CFD technique analyze the influence of the calender roll structure on the surface temperature and cooling effect in the development of the calender roll, which provided the basis for the design of the glass forming process and the design improvement of the calendering roller structure, thus improving the production efficiency and product quality, has an important help inreducing the development cost of calendering roll and improving the post-molding process, it is the only way for the technological innovation and the leap development of the rolling glass production enterprises.

\section{Process Design}

In the forming process of calendering glass, the surface temperature of calender roller is the key factor to affect the quality of finished products. The factors that affect the heat transfer include the structure of the forming roller, the cooling water temperature and the inlet velocity. In this paper, based on the forming roll structure used in the actual production, the construction of different roller structure. Based on the basic theory of computational fluid dynamics (CFD), using Reynolds equation method, can realize the turbulence model and wall function method, establishes the mathematical model of the cooling water heat exchanger in different cavity flow structure of calender roll [3]. The model was simulated numerically using ANSYS FLUENT software under the same process conditions to simulate a rolling cycle from contact to leaving the molten glass and then to the next contact with the molten glass. The effect of the structure of the cooling water channel on the heat transfer performance of the calender was analyzed by the software post - processing results. The program flow is shown in Figure 1. 


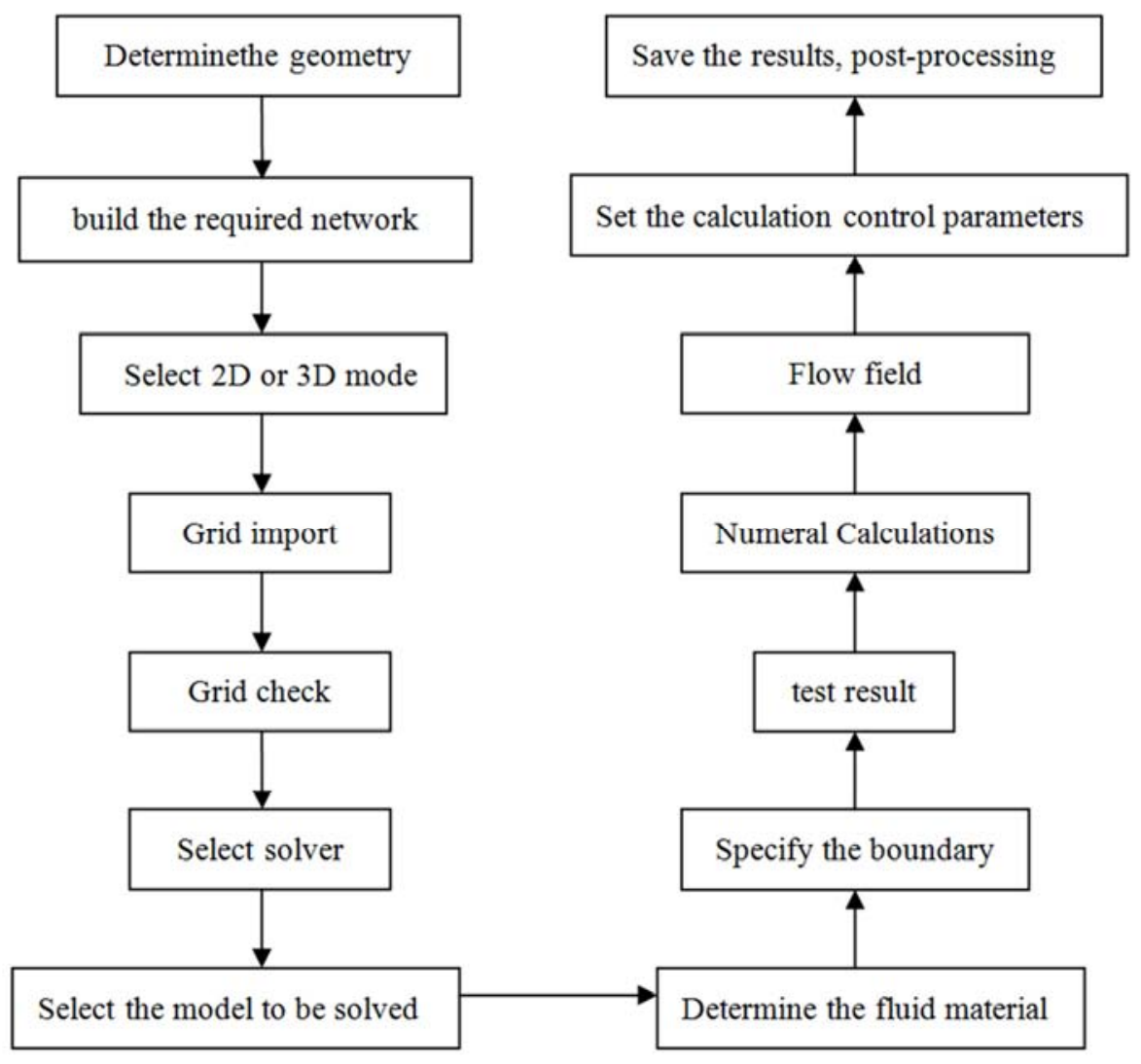

Figure 1. FLUENT Solving general steps.

\section{Calender Roll Structure Design}

\subsection{Brief Design}

The calender roll is the main equipment for glass calendering, which is mounted on the calender by the drive device to drive the molten glass flow through the upper and lower rollers and cool through the cooling water in the roll to form the plate. The calender roll is composed of a roller body and a roller shaft. The glass calender roll used in this study is formed by an outer roller, an inner roller and a roller shaft, and the cavity is divided into four separate cavities through a sealing partition board. As figure 2 .

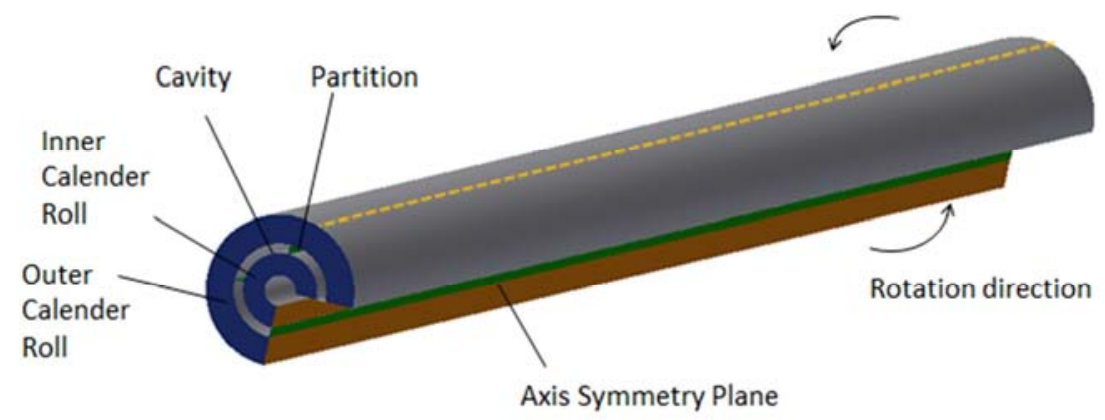

Figure 2. Schematic diagram of the axial profile of the calender roll.

During the rolling process, the cooling water is constantly flowing from one end of the four cavities to the other end. This one-way flow pattern has a simple structure, but to ensure uniformity of the surface temperature of the calender roll, it is necessary to greatly increase the flow rate of the cooling water [4]. The outer surface of the outer roll is in contact with the molten glass, and the heat-resistant alloy steel is generally used. In order to increase the sunlight transmittance of the ultra-white glass, the surface of the roller is subjected to engraving, and in order to increase the service life of the calender roll. The outer surface of the outer roller must also be chrome-plated.

The basic dimensions of the roller are as follows: Axial length of $2400 \mathrm{~mm}$, inner roller $50 \mathrm{~mm}$, inner roll diameter of $120 \mathrm{~mm}$, inner roller radial thickness of $35 \mathrm{~mm}$, outer roller diameter of $240 \mathrm{~mm}$, outer roll radial size $45 \mathrm{~mm}$, cavity radial size $15 \mathrm{~mm}$. Since the cooling water in the chamber flow area is an axial flow, consider the comparison of the normal roll 
diameter and the roll diameter structure with the knots. The depth of the nodal is $5 \mathrm{~mm}$. In order to observe the effect of roller diameter structure on the flow and heat transfer performance.

\subsection{Model Establishment}

Three model structures were constructed in the study, as shown in the figure. The structure of the smooth roller is the actual structure of the structure, with a wave structure of the model structure is: the heat transfer between the fluid and the wall is achieved through the boundary layer. The flow condition in the boundary layer has a giant influence on the heat transfer between the fluid and the wall. In the laminar boundary layer, heat transfer is mainly done by means of heat conduction. This heat transfer and heat convection heat transfer effect compared to the effect is much worse. In most of the turbulent boundary layer, heat transfer is carried out by turbulent mixing of this intense convective heat transfer, so that the heat transfer effect of turbulent fluid is much better than the laminar fluid. However, the heat transfer in the laminar flow is mainly based on the thermal conductivity. The main factors affecting the heat transfer are the thermal resistance of the laminar bottom, the thinner the laminar flow, the smaller the thermal resistance between the fluid and the wall, and the heat transfer process. Therefore, to set the artificial roughness of the boss structurein the channel, to enhance the fluid disturbance, reducing the laminar flow layer thickness, so as to achieve enhanced fluid and wall convection heat transfer purposes $[5,6]$.

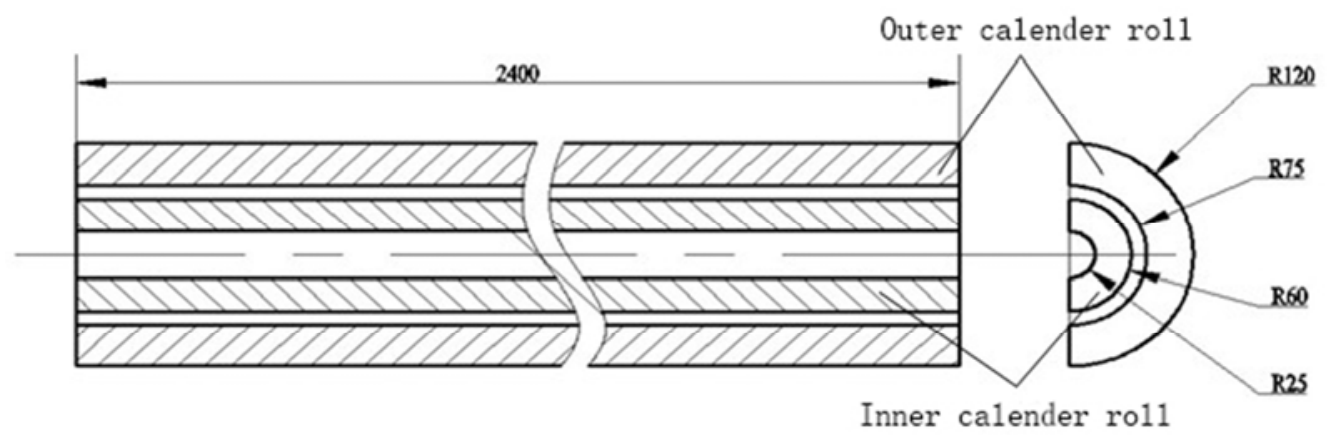

Figure 3. Geometric model of smooth roller.

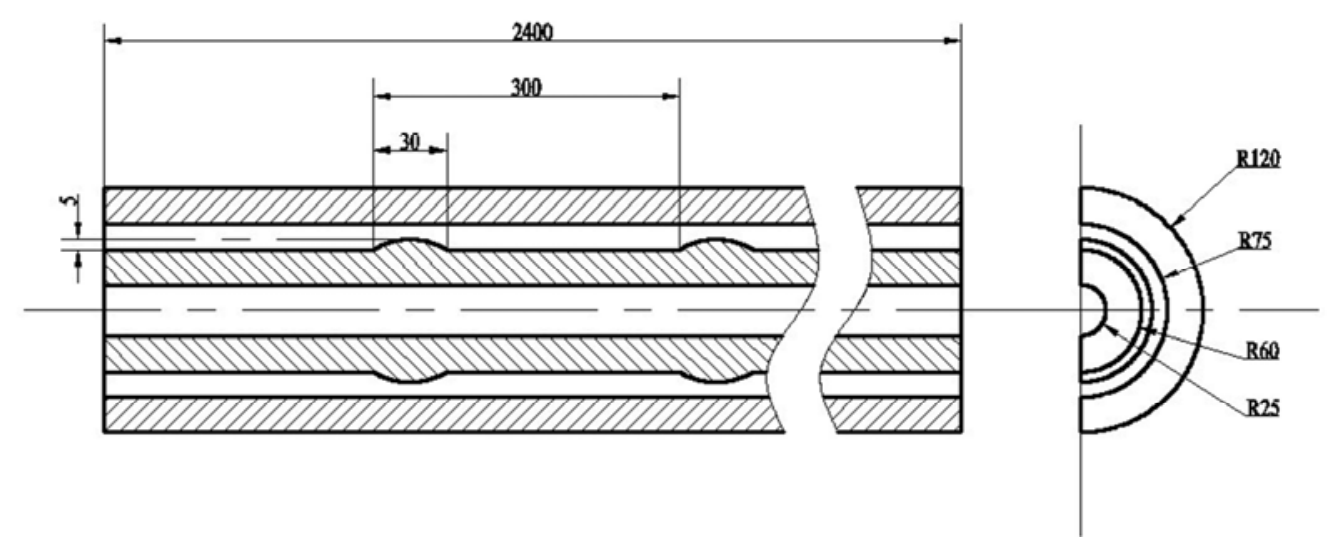

Figure 4. The inner roller has a node struct.

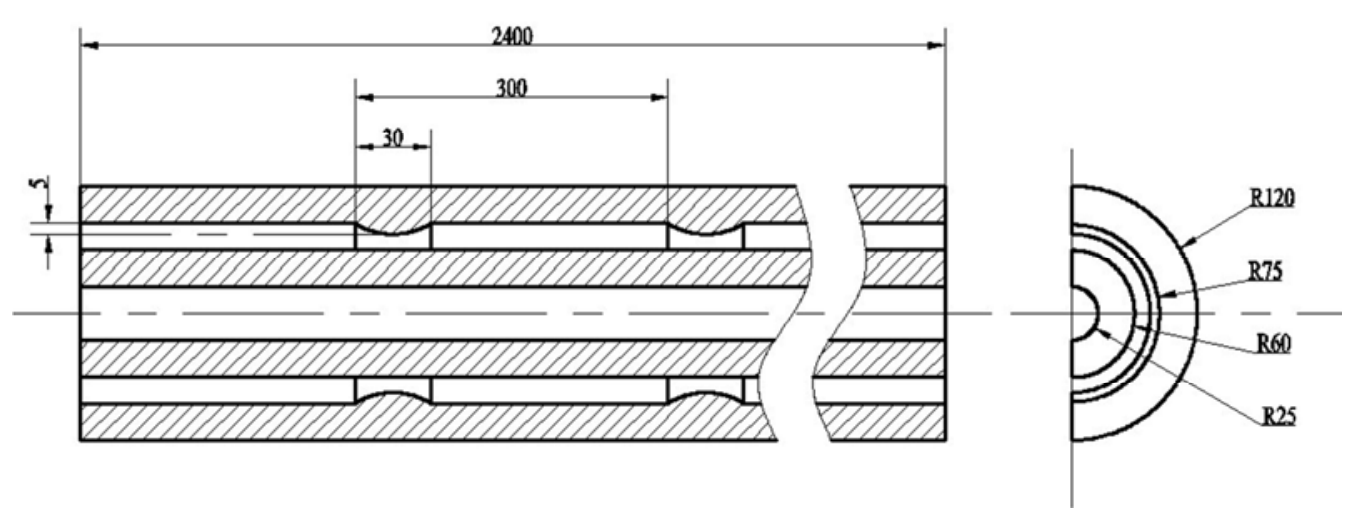

Figure 5. The outer roller has a node structure model. 


\section{FLUENT Calculation Parameter Settings}

(1) Control equation:

Because the glass is very thin, can only consider the heat conduction along the thickness direction, ignoring the internal heat conduction products, The heat transfer of the calendering roller is also carried out in this way, in the thickness direction for local transient thermal analysis can be, the temperature field control equation:

$$
\rho C \frac{\partial T_{P}}{\partial \mathrm{t}}=\frac{\partial}{\partial \mathrm{s}}\left(K_{\mathrm{e}} \frac{\partial T_{P}}{\partial \mathrm{s}}\right)
$$

In type:

$T_{P}$ - temperature, ${ }^{\circ} \mathrm{C}$;

$K_{\mathrm{e}}$ - equivalent thermal coefficient;

$\mathrm{S}$ - local coordinates along the thickness direction.

The cooling water flow inside a roller are controlled by the physical conservation laws, In order to simulate the flow and heat transfer, we must first establish the mathematical relationship between the various variables describing the problem, that is, to establish the mathematical model of fluid flow and heat transfer. In this paper, we must establish the mathematical model of fluid flow and heat transfer. In general, in the area under consideration, the flow and heat transfer of the fluid should satisfy the mass, momentum and energy conservation equations: 1) mass conservation equation; 2) momentum conservation equation; 3) energy conservation equation $[7,8]$;

(2) Physical model: Select the standard $k-\varepsilon$ equation model in the Viscous turbulence model.

(3) Material Properties Settings: For Fluid, select water-liquid from FLUENT's own database to create material properties; and Solid select the properties of the steel material and create it.

(4) Domain conditions selection: roller inside and outside the diameter of the Solid area, cooling water channel for the Fluid area.

(5) Boundary conditions: the entrance for the entrance speed boundary conditions, the size of $0.5 \mathrm{~m} / \mathrm{s}$, water temperature $20^{\circ} \mathrm{C} \quad(295 \mathrm{k})$; export pressure boundary conditions, the pressure for a standard atmospheric pressure; Symmetric surfaces are symmetry; the others are wall boundary types. The outer surface temperature of the roller is 1100 degrees centigrade.

\section{Simulation Results and Analysis of FLUENT}

\subsection{Velocity Field and Velocity Vector Analysis}

In the simulation results, he velocity field and velocity vector diagram are used to showin different calendering rolls, the flow of the cooling water fluid in the chamber is further analyzed and its effect on heat transfer is further analyzed from the flow conditions.

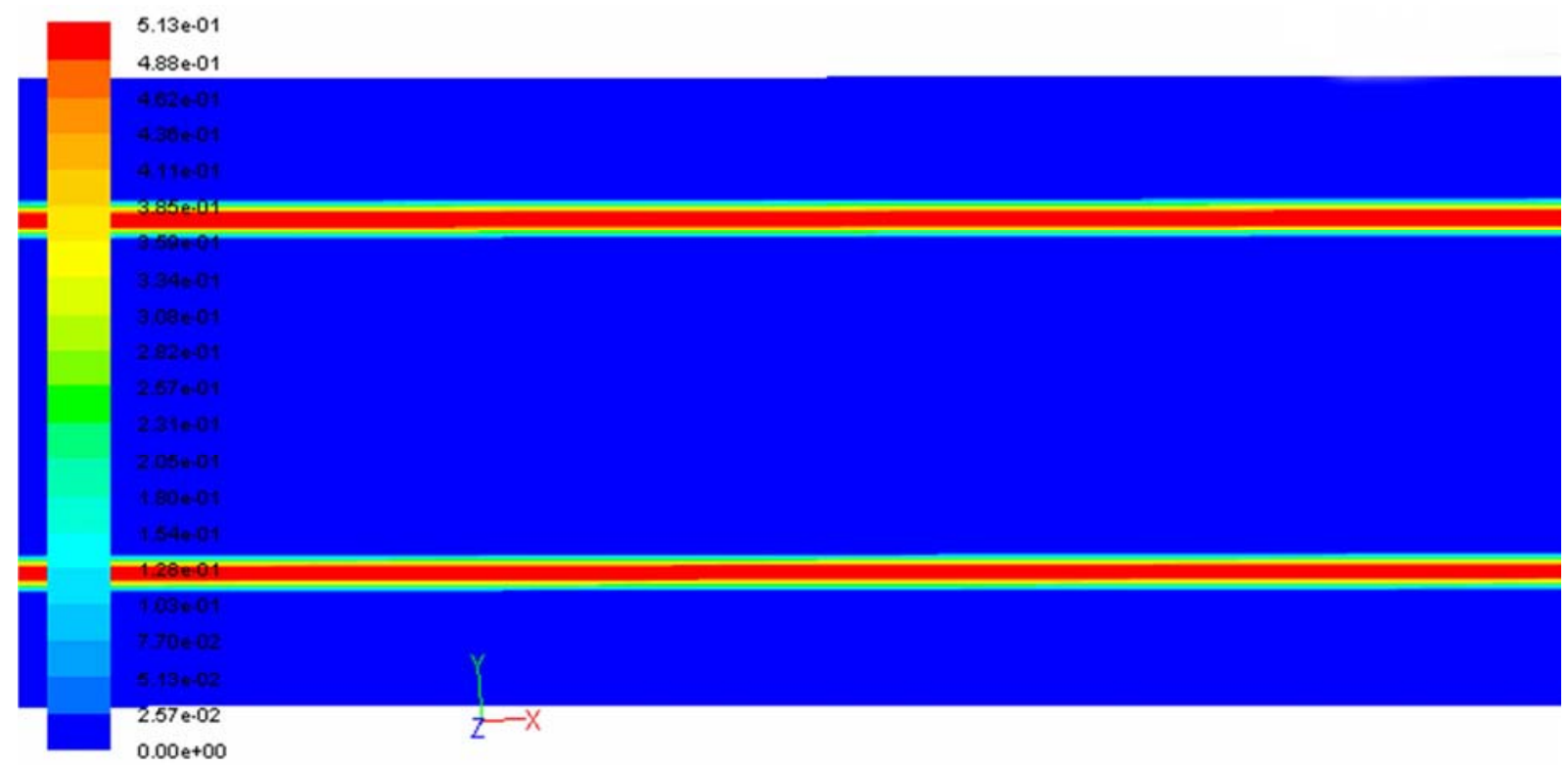

Contours of Velocity Magnitude (m/s) 


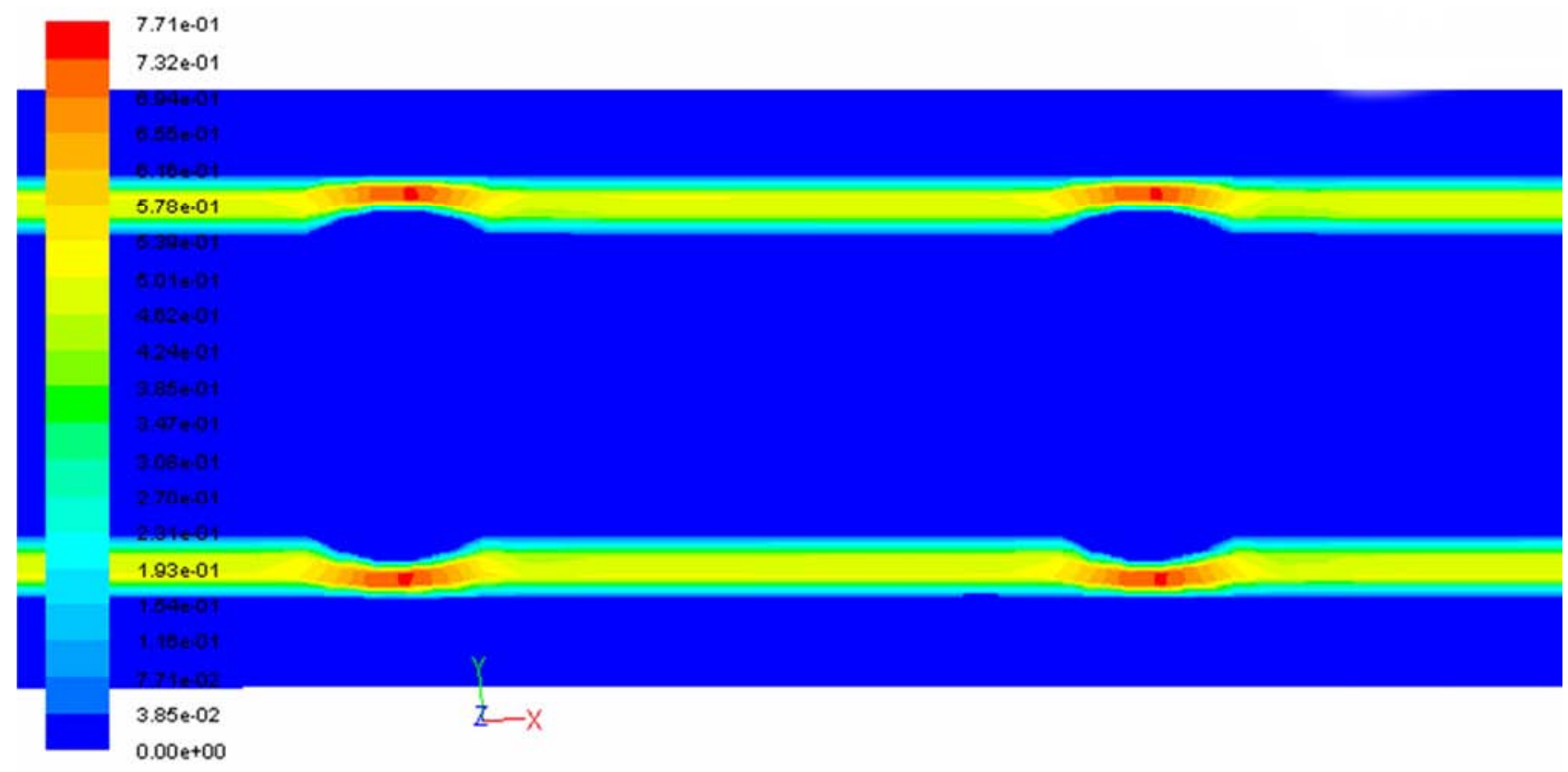

Contours of Velocity Magnitude (m/s)

Figure 7. Within the roller with a symmetrical section of the structure of the full development of the speed of the cloud.

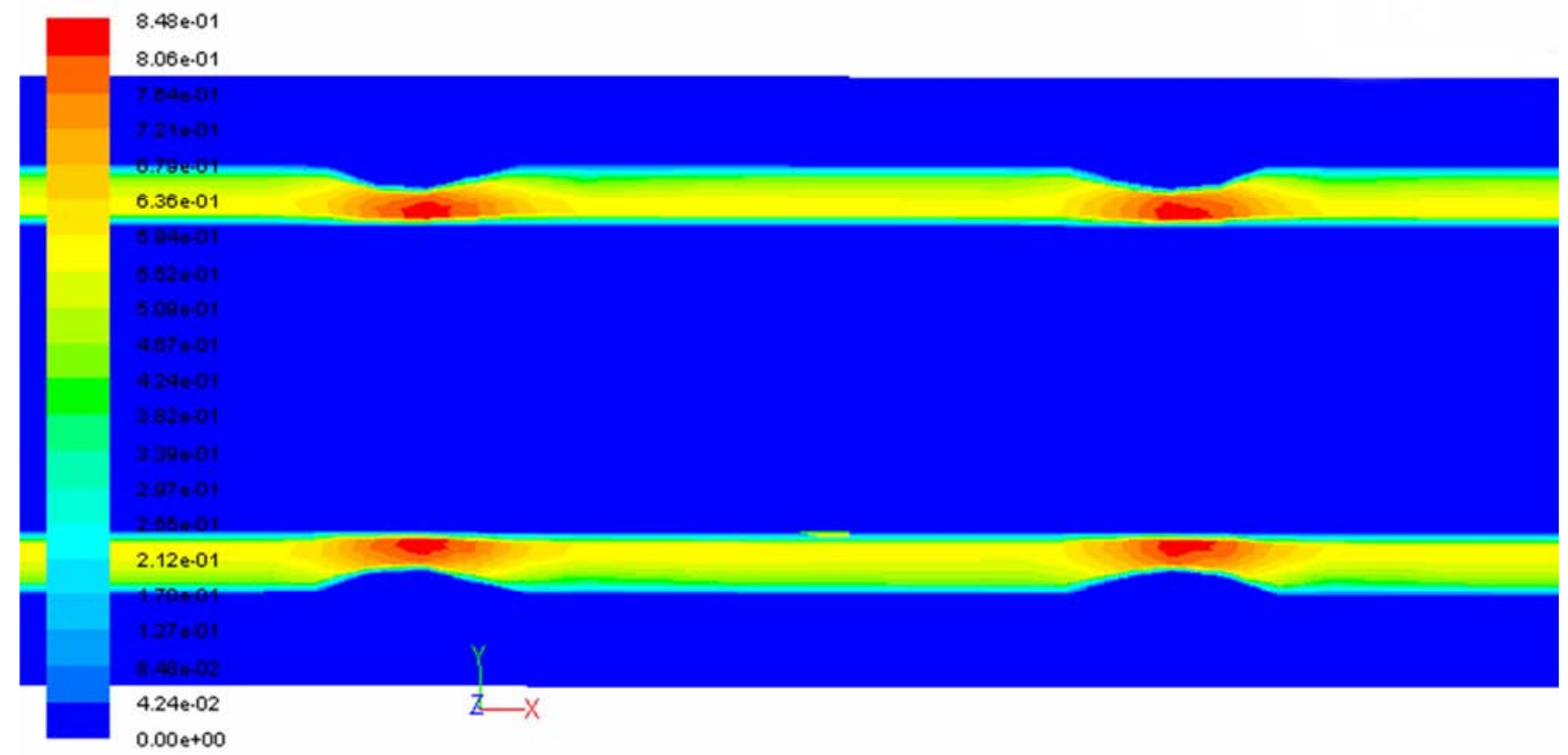

Contours of Velocity Magnitude (m/s)

Figure 8. The outer roller has a node structure symmetrical cross section full development speed cloud.

Figure 8 shows a partially enlarged view of the smooth roller structure. It can be seen from the figure that the velocity gradient in the normal direction of the wall is gradually reduced to zero at a very thin distance near the wall. This is basically consistent with the description of boundary layer theory in fluid mechanics [9], which flows almost in the direction of the wall. It can be seen from Fig.9 that the thickness of the boundary layer of the circular arc segment is significantly smaller than that of the straight pipe section, which indicates that the velocity of the fluid decreases at the nodules, the static pressure increases, increases the heat transfer, and the design with the node structure Improved heat transfer of the wall. 


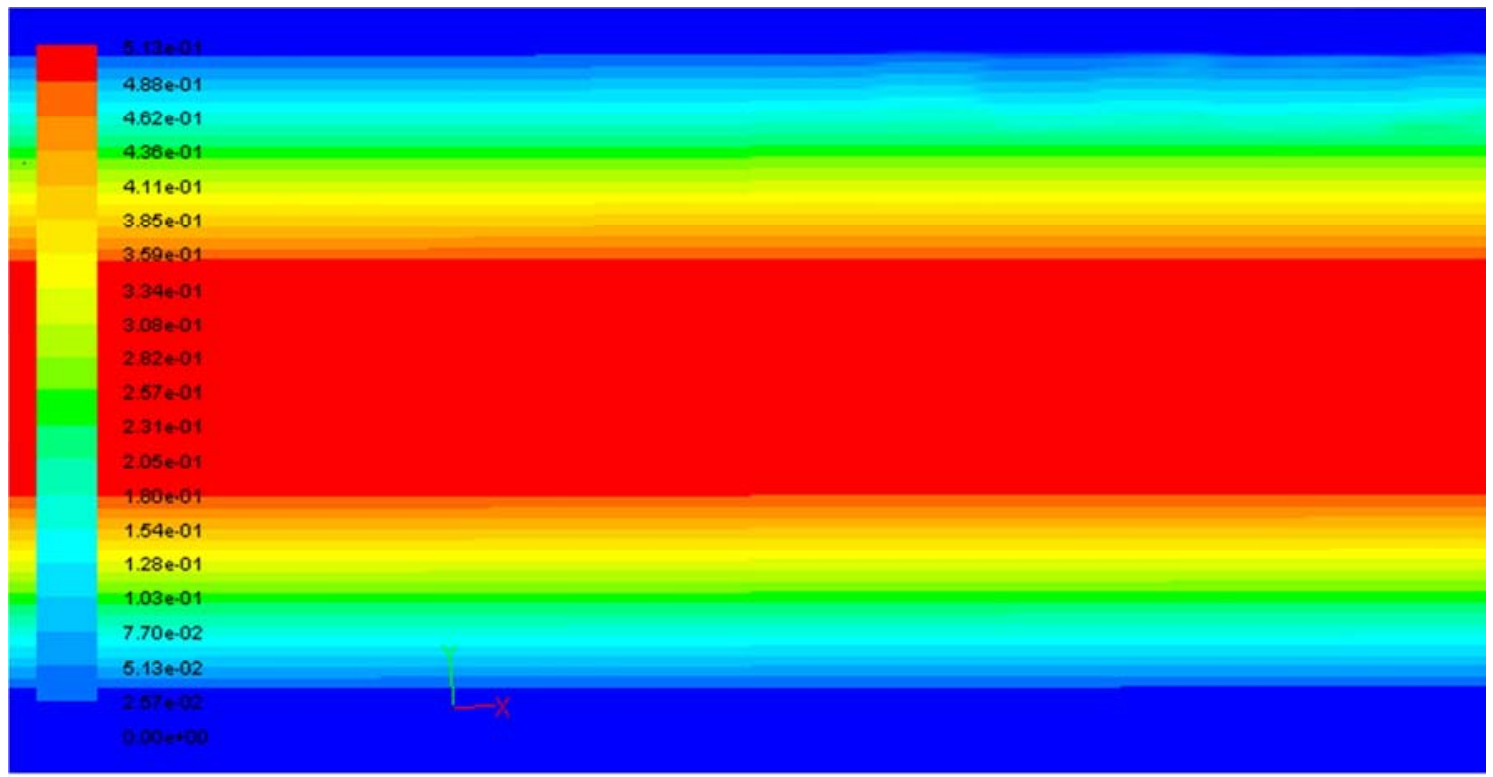

Contours of Velocity Magnitude (m/s)

Figure 9. Smooth roller rolls partially enlarged view of the velocity boundary wall.

Figure 6 8 shows the smooth calendering roller and the inner and outer band structure of the calendar roller on the symmetrical cross section of the speed of the cloud, The blue area shown in the diagram is the solid area of the calender roll, and the other color is the area of the cooling water flow, As can be seen from the comparison of the velocity cloud image: 1) smooth calendering roller structure to fully develop the mainstream of the fluid velocity changes little, the flow is very simple. As shown in Figure 6, in the case of a given inlet velocity of $0.5 \mathrm{~m} / \mathrm{s}$, the maximum velocity of the main flow region is $0.513 \mathrm{~m} / \mathrm{s}$, which is the same as that of the given inlet velocity, which is due to the uniformity of the cooling water channel structure; 2) increased the turbolator wave structure in the mainstream area day maximum speed appears red position in the wave section "arc", in Figure 7 the maximum speed of up to $0.771 \mathrm{~m} / \mathrm{s}$, Figure 8 maximum speed up to $0.848 \mathrm{~m} / \mathrm{s}$, the velocity field in the nodal structure exhibits a periodic distribution. When the fluid is composed of "straight pipe section" - "circular section" - "straight pipe section", the flow path section is periodically contracted and expanded to form periodic interference, so that the flow rate also shows a cyclical changes with the cross-section, the flow section of the straight pipe at the speed of the basic changes little.

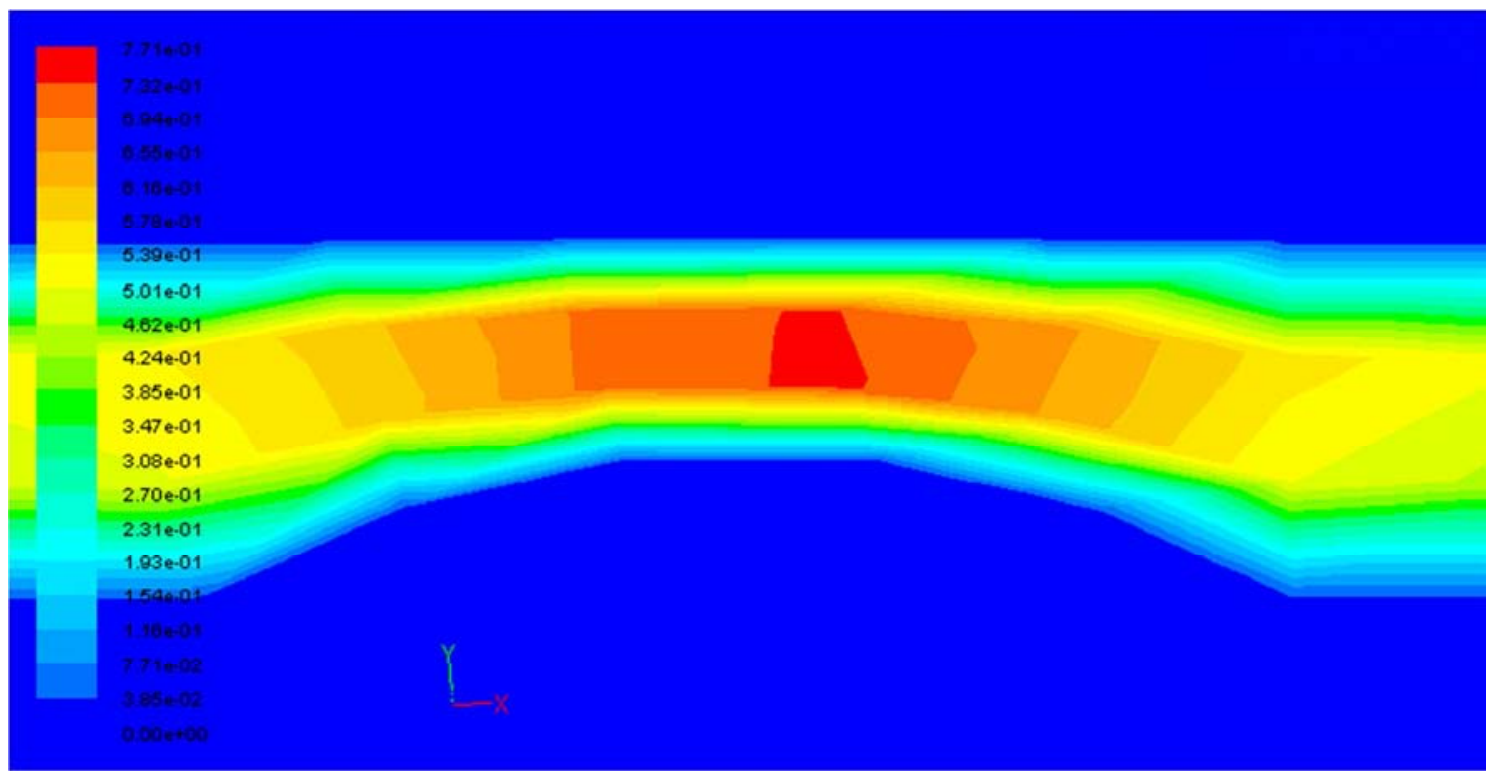

Contours of Velocity Magnitude (mis) 


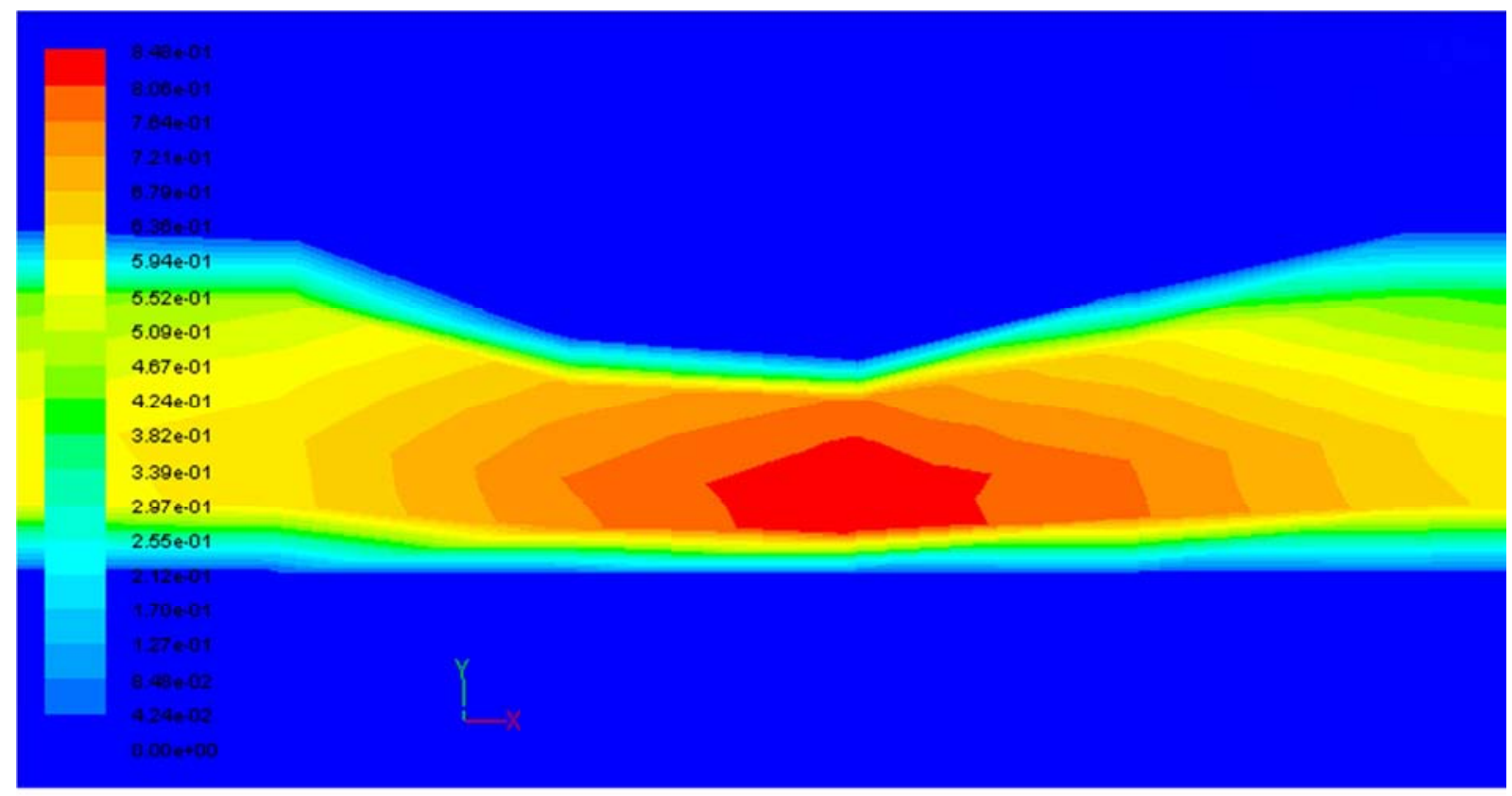

Contours of Velocity Magnitude (m/s)

Figure 10. Inside and outside thecalendering roller has a section of the speed of the local structure.

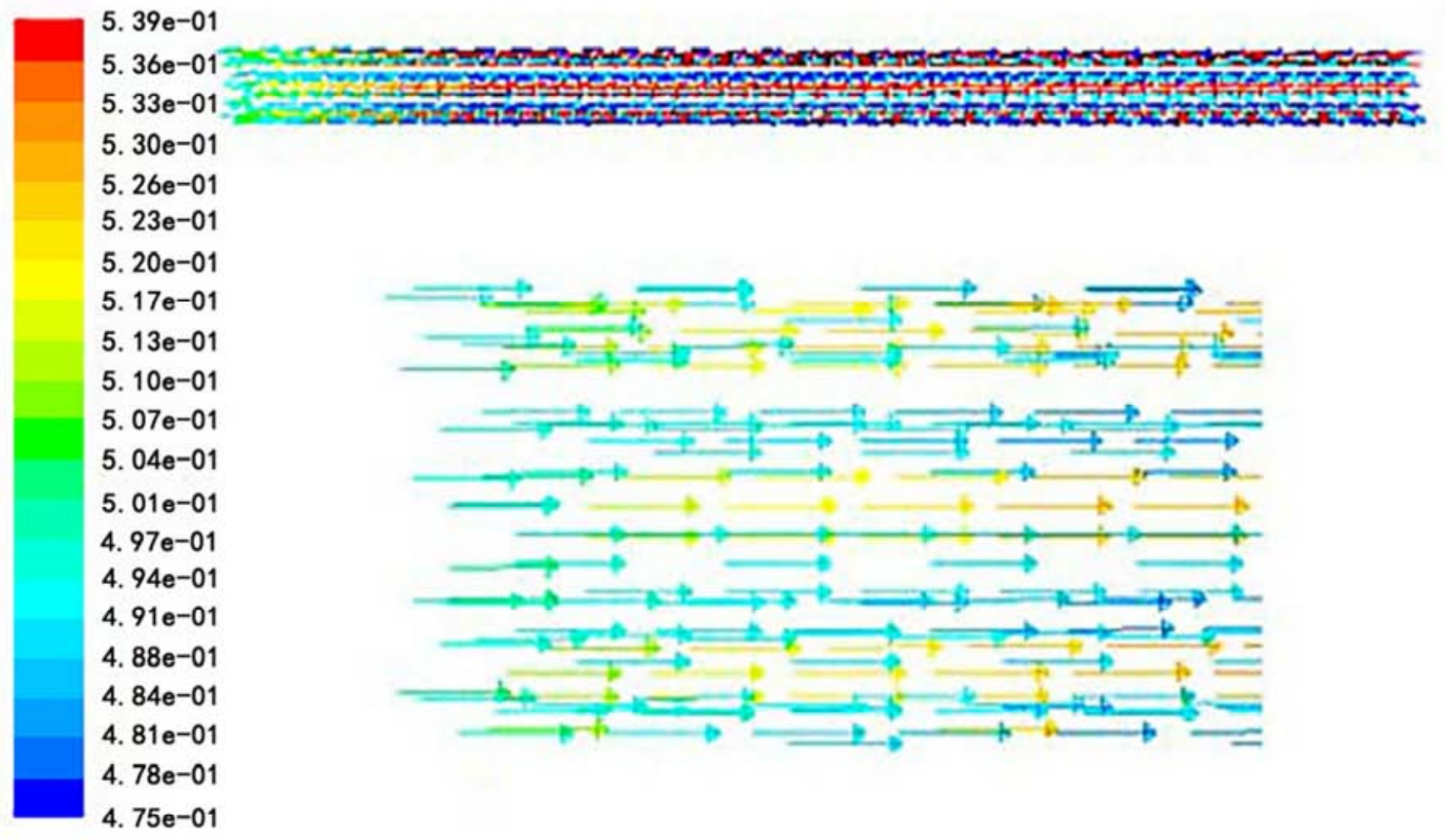

Velocity Vectors Colored By Velocity Magnifude $(\mathrm{m} / \mathrm{s})$

Figure 11. Velocity vector diagram of symmetrical section section of smooth calender roll. 


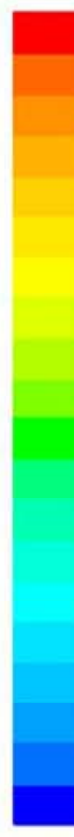

7. $98 \mathrm{e}-01$

7. $78 \mathrm{e}-01$

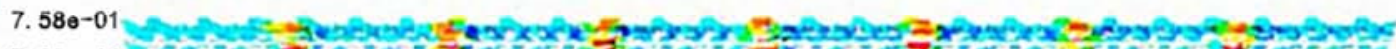

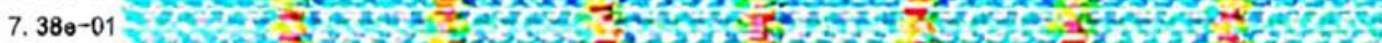

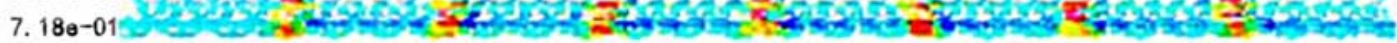

6. $98 \mathrm{e}-01$

6. $78 \mathrm{e}-01$

6. $58 \mathrm{e}-01$

6. $38 \mathrm{e}-01$

6. $18 \theta-01$

5. $98 \theta-01$

5. $78 \theta-01$

5. 58e-01

5. $38 \mathrm{e}-01$

5. $18 \theta-01$

4. $98 \theta-01$

4. $78 \mathrm{e}-01$

4. $58 \mathrm{e}-01$

4. $38 \theta-01$

4. $18 \mathrm{e}-01$

3. $98 \mathrm{e}-01$

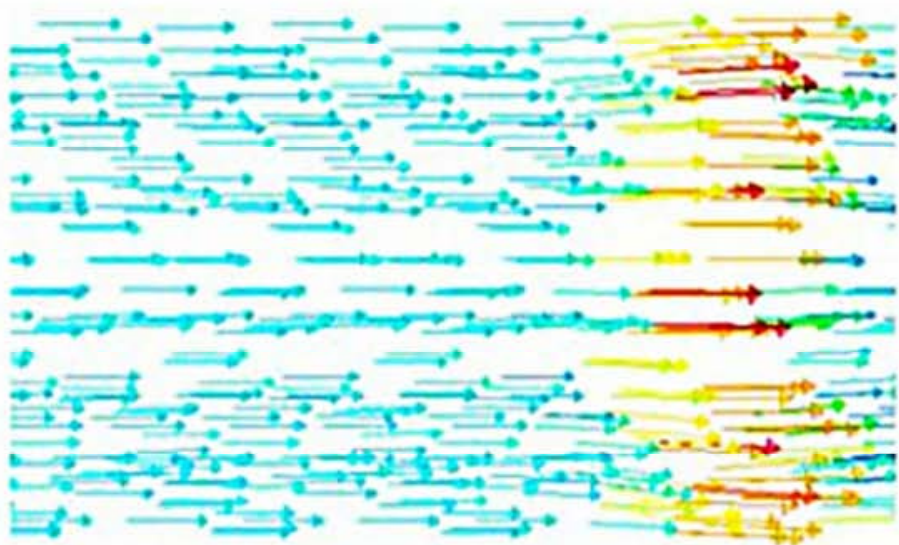

Velocity Vectors Colored By Velocity Magnifude (m/s)

ANSYS FLUENT 12.0 (3d.pbns, ske)

Figure 12. The calender roll has a nodal structure velocity vector

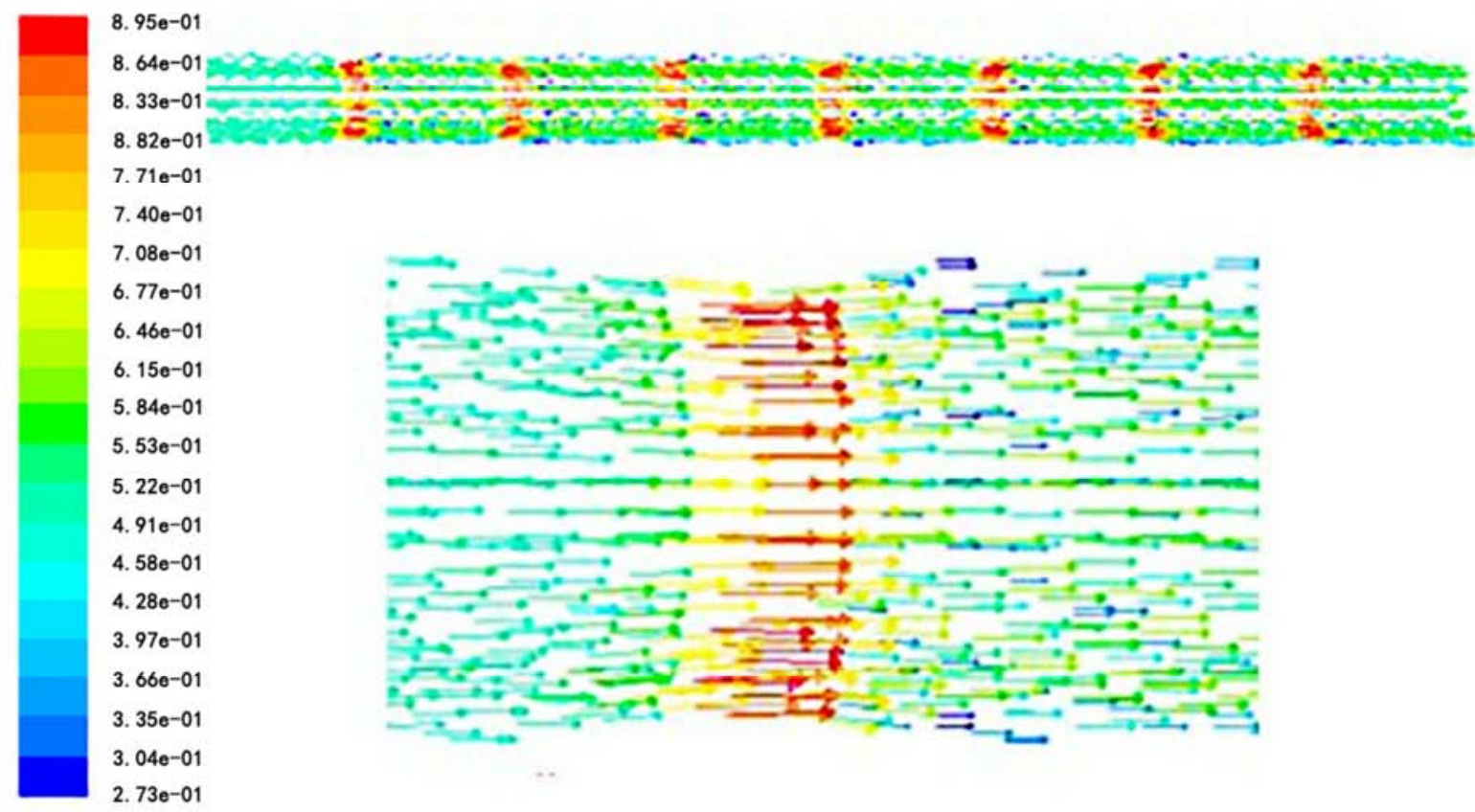

Velocity Vectors Colored By Velocity Magnifude(m/s)

ANSYS FLUENT 12.0 (3d. pbns, ske)

Figure 13. The outer calender roll has a nodal structure velocity vector

The flow mass in the cross section of the cooling water flow channel is changing at any time in addition to the flow in the axial direction, as shown in the vector diagram of the various calendering structures shown in Figs. 11 to 13. The flow velocity of the fluid in the light roller structure is relatively large at the central part, and the flow velocity increases suddenly after the inlet section, so that the heat in the whole structure of the whole structure is more heat, and 
the heat transfer effect is obvious. The heat will be less uniform, which is consistent with the actual forming process of heat transfer characteristics. In addition to the wave velocity of the wave segment, the other velocity of the fluid is constant, and the flow velocity of the outer roller with the nodal structure is larger than that of the inner roller.

\subsection{Nu Number Analysis}

Figures 14 and 15 show the Nu number of the wall of the nodal structure with the smooth calendering roller and the outer calender roll. Nu number is the convective heat transfer coefficient of the dimensionless, enhanced heat transfer performance can be directly reflected by the number of $\mathrm{Nu}$ number [10]. It can be seen from the figure: 1) The $\mathrm{Nu}$ number at the entrance is relatively large, indicating that the heat transfer between the fluid and the roll wall, from the entrance, the boundary layer is gradually increased from zero, that is, the entrance of the boundary layer relative to the full development of the So that the local surface heat transfer coefficient of the inlet section is higher (inlet effect). In addition to the entrance effect, the entrance of the water because of the lower temperature and with the cooling water in the channel flow has to take away part of the heat transfer, so the entrance of the convection heat relative to the full development of strong. The $\mathrm{Nu}$ number of the node structure is significantly larger than the smooth diameter structure. 2) The Nu number of each arc segment with the node structure is periodically changed, and the distribution regularity is basically the same. With the gradual increase in the temperature of the cooling water, the $\mathrm{Nu}$ number gradually decreases along the flow direction, so that the local heat transfer can also be reflected by every node unit. Experimental studies show that when the fluid is turbulent flow, the inlet section is not affected by the average surface heat transfer coefficient as long as $1 / \mathrm{d}>60$ [11].

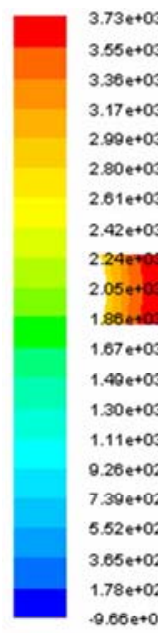

Contours of Surface Nusselt Number

ANSYS FLUENT 12.0 (3d, pbns, ske)

Figure 14. Smooth calenderingroller with node structure Nu number distribution.

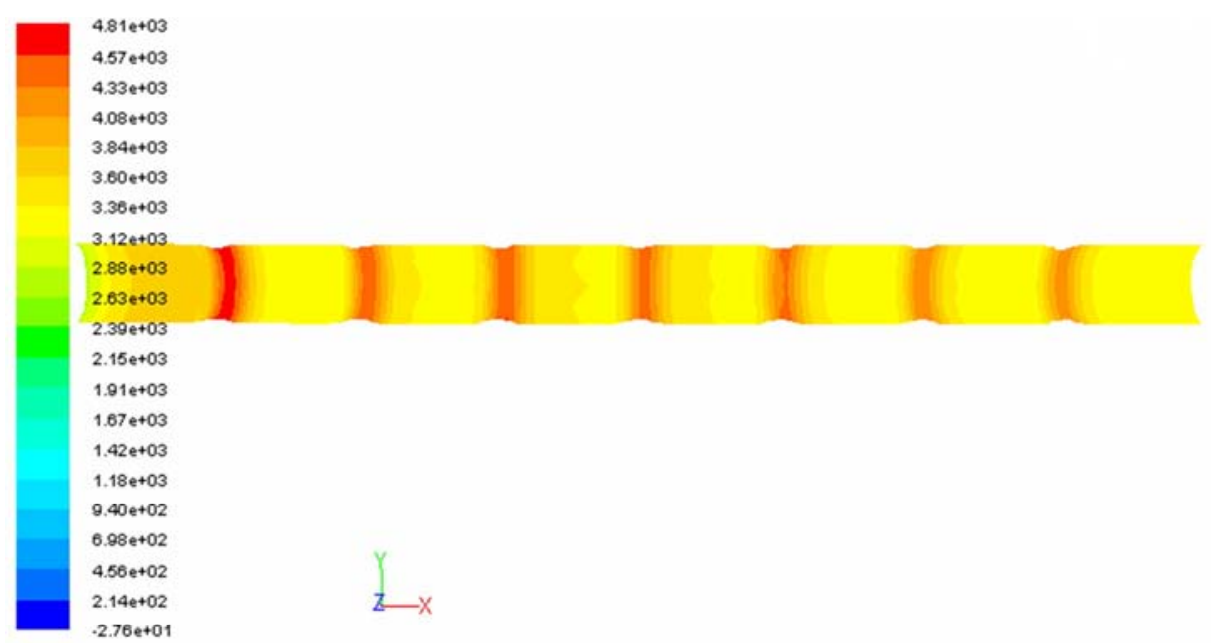

Contours of Surface Nusselt Number

Figure 15. The outer calendering roll has a node structure Nu number distribution. 


\subsection{Temperature Field Analysis}

Since the size of the cooling water channel is small for the entire model, only to consider the impact of the calendering structure on the heat transfer, given the inlet flow velocity is low, so the temperature change is not large, from the temperature cloud diagram to see the rolling roller structure of the temperature change is not obvious. As shown in Figure 20 , it is a partial magnification cloud near the exit at the symmetrical section. It can be seen from the figure that when the temperature from the top to the inner calender rolls changes from high to low, the cooling water takes away part of the heat during the flow of the calendering roll, causing the cooling water to rise, so that the temperature of the outer wall of the roll is higher than the temperature in the front. The results of the simulation under three different calendering roll diameters are shown in Table 1.

Table 1. Simulation conditions and results.

\begin{tabular}{|c|c|c|c|c|}
\hline Calendering roller structure & $\begin{array}{l}\text { Average temperature of } \\
\text { inner surface in } \\
\text { calender roll }\left({ }^{\circ} \mathrm{C}\right)\end{array}$ & $\begin{array}{l}\text { The average temperature } \\
\text { of outer surface } \\
\text { incalender roll }\left({ }^{\circ} \mathrm{C}\right)\end{array}$ & $\begin{array}{l}\text { Calendering coefficient } \\
\text { of inner surface of } \\
\text { calender roll }\left(\mathrm{w} / \mathbf{m}^{2} \cdot \mathbf{k}\right)\end{array}$ & $\begin{array}{l}\text { Calendering coefficient } \\
\text { of outer surface of } \\
\text { calendar roll }\left(\mathrm{W} / \mathrm{m}^{2} \cdot \mathbf{k}\right)\end{array}$ \\
\hline Smooth calendering roller & 56.8 & 1031 & 2005.7 & 235.1 \\
\hline The outer calender roll has a wave & 54.4 & 1028.3 & 2128.97 & 302.3 \\
\hline
\end{tabular}

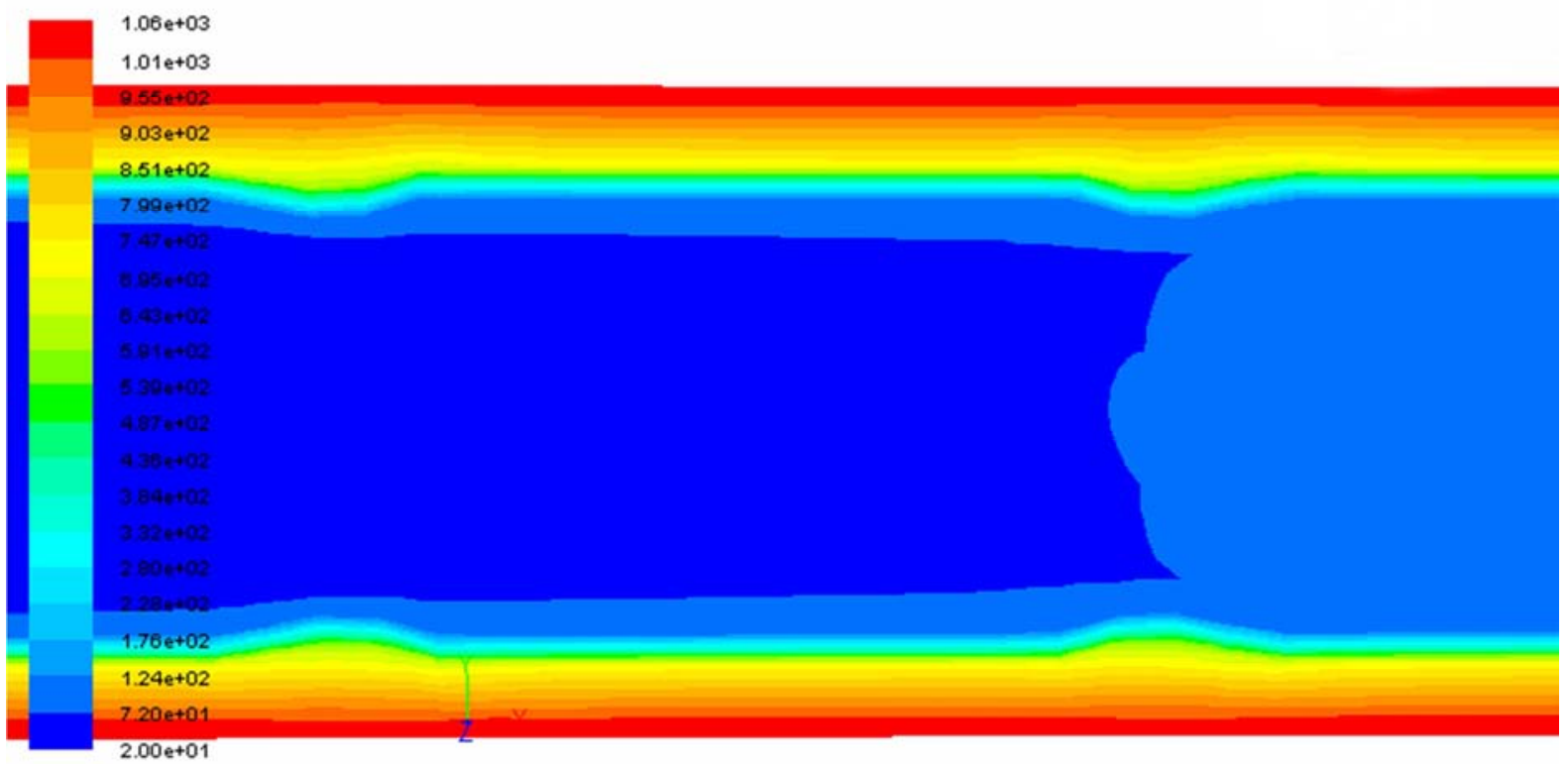

Contours of Total Temperature (c)

Figure 16. The outer calender roll has a node structure with a local amplification temperature field.

Compared with the velocity field and the temperature field, it can be seen that the temperature of the hot fluid is high in the region where the fluid velocity is high, and the velocity field and the temperature field are mutually affected [12]. The increase of the fluid velocity is beneficial to the convective heat transfer and the change of the temperature field of the increase, to promote the turbulence of the fluid, enhanced convection heat transfer, so that the temperature field changes. It can also be seen from Table 1 that the average temperature of the inner and outer surfaces of the roll with the increase of the nodal structure is lower than that of the smooth calendering roller, and the heat transfer coefficient of the inner and outer roller surfaces Higher than the smooth calendering roller structure, indicating that with a node structure is conducive to enhanced heat transfer.

\section{Conclusion}

Constructing different geometrical model of calendering roll diameter. The effect of roll diameter structure on the heat transfer effect is analyzed from the velocity field and temperature field by FLUENT software.

(1) Through the analysis of the model structure, the different geometric models of the calendering roller are constructed, and the meshing method of the structured hexahedral mesh in GAMBIT is used to divide the mesh. After the detection of the grid quality satisfies the requirement, The method of division can reduce the computational complexity and improve the computational efficiency under the premise that the calculation result is reasonable. 
(2) By analyzing the velocity field, velocity vector and local $\mathrm{Nu}$ number, the increase of the nodal structure will increase the flow rate of the cooling water, thus strengthening the heat transfer. This is the same as the results of the current study [13] on the study of wave tube heat exchangers;

(3) Through the analysis of the temperature field of different calendering rolls, it is known that the overall temperature of the calender roll is lower than that of the optical roller, and the heat transfer coefficient on the inner and outer surfaces is larger than that of the optical roller. It is shown that the increase of the nodal structure is beneficial to the heat transfer, and the heat transfer effect of the calender roll with wave junction rolling roller structure is relatively good.

\section{Acknowledgements}

The authors would like to acknowledge financial support from the National Natural Science Foundation of China (Grant No. 51375143) and the Talents Project of Universities of Henan Province (2015RCJH12).

\section{References}

[1] Cuce E, Young C H, Riffat S B. Performance investigation of heat insulation solar glass for low-carbon buildings [J]. Energy Conversion \& Management, 2014, 88: 834-841.

[2] J. Eisenträger, K. Naumenko, H. Altenbach, et al. A user-defined finite element for laminated glass panels and photovoltaic modules based on a layer-wise theory [J]. Composite Structures, 2015, 133: 265-277.

[3] $\mathrm{Wu}$ Zhen-zhen. Finite element analysis of the process of photovoltaic glass calendering [D]. Henan University of Technology, 2015.
[4] Zhao D Q, Tang Y, Huang Z G. Glass Toughening System Modeling and Simulation of Flow Field Based on Fluent [J]. Mechanical \& Electrical Engineering Technology, 2016.

[5] Isaev S A, Leontiev A I, Baranov P A, et al. Numerical Simulation of the Intensification of the Heat Exchange in a Plane-Parallel Channel with a Cylindrical Shallow Dimple on the Heated Wall [J]. Journal of Engineering Physics \& Thermophysics, 2016, 89(5): 1186-1201.

[6] Geng Tie, Yan Liqun. On the research of heat transfer enhancement technology of artificial roughness. [J] Machinery Design \& Manufacture 2011, (02): 252-253.

[7] Fang Qiang. Production Technology, Equipment and Practice of Solar Ultra - white Glass [J]. 2007 Proceedings of China Float Glass and Glass New Technology Development Seminar, 2010: 29-33.

[8] Bei Mu. Photovoltaic glass production process [J]. Display device technology, 2010, 2: 1-2.

[9] Ledoux M, Hami A E. 1. Mechanics and Fluid [M] // Fluid Mechanics: Analytical Methods. John Wiley \& Sons, Inc. 2017.

[10] Cao Yingjia. FLUENT for Corrugated tube Heat Exchanger [D]. Wuhan University of Technology, 2012.

[11] Lu X Y, Li X G, Liu J M, et al. Numerical Simulation of Flow Fluid in Elbow Pipe Based on FLUENT and the Establishment of the Pressure Model [J]. Applied Mechanics \& Materials, 2015, 713-715: 39-42.

[12] Li L, Du X, Zhang Y, et al. Numerical simulation on flow and heat transfer of fin-and-tube heat exchanger with longitudinal vortex generators $[\mathrm{J}]$. International Journal of Thermal Sciences, 2015, 92: 85-96.

[13] Bhattacharyya S, Chattopadhyay H, Bandyopadhyay S. Numerical Simulation of Fluid Flow and Heat Transfer Enhancement in a Circular Wavy Channel [J]. Advances in Mechanical Engineering (Hindawi Publishing Corporati, 2015, 2013(6): 1104-1116. 